\title{
Operative Instandhaltung im Zeitalter der Digitalisierung
}

\author{
S. Sade ${ }^{1}$, T. Bartsch ${ }^{2}$, F. Albers ${ }^{3}$, D. Kowalke ${ }^{4}$
}

\section{Zusammenfassung}

Im Rahmen des Forschungsprojektes „Milling-Waterjet“ an der Technischen Hochschule OstwestfalenLippe wurde ein operatives Instandhaltungswerkzeug entwickelt, welches die nach der DIN 31051 beschriebenen Maßnahmen zur Instandhaltung in digitaler Form abbildet. Das Anwendungsobjekt in diesem Fall ist eine Wasserstrahlschneidanlage. Der vorliegende Beitrag beschreibt das digitale Instandhaltungswerkzeug.

\section{Stichwörter}

Diagnose, Digitalisierung, Fehlerbaum, Instandhaltung, Wasserstrahlschneiden

\section{Einleitung}

Nach der DIN 31051 wird unter Instandhaltung die Kombination aller technischen und administrativen Maßnahmen sowie Maßnahmen des Managements während des Lebenszyklus eines Objektes verstanden, die dem Erhalt oder der Wiederherstellung ihres funktionsfähigen Zustands dienen, sodass es die geforderte Funktion erfüllt [1].

Der Prozess des Wasserstrahlschneidens ist technologisch anspruchsvoll. Aus diesem Grund bedarf es einer intensiven Instandhaltung. Von regelmäßig anfallenden Wartungsmaßnahmen, bis hin zur außerplanmäßigen Instandsetzung, welche den funktionsfähigen Zustand wiederherstellen, treten diverse Handlungsmaßnnahmen im Laufe des Lebenszyklus der Anlage auf.

Um sich Hilfe bei der Umsetzung dieser Maßnahmen zu verschaffen, musste sich der Bediener der Anlage in der Vergangenheit an Handbüchern in Papierform und an seinem Erfahrungswissen bedienen. Weiterhin gab es die Möglichkeit am Erfahrungsschatz von anderen Mitarbeitern teilzuhaben. Damit eine zukunftsorientierte Instandhaltung an der Anlage möglich ist, bei der das Wissen über die Instandhaltung dieser gebündelt wird, wurde im Rahmen des Forschungsprojektes eine Diagnoseanwendung entwickelt, in der die Technische Hochschule OWL als Pilot-Anwender fungiert [4]. Die Umsetzung erfolgte mit der Software „QuickSteps“ [2].

In Bild 1 wird der Aufbau der Wasserstrahlschneidanlage gezeigt. Dazu wurde die Werkzeugmaschine in Funktionseinheiten hierarchisch gegliedert. Bild 2 veranschaulicht den Hochdruckschneidkopf der Anlage im laufenden Betrieb. Die Instandhaltung des Schneidkopfes hatte für die Entwicklung der Diagnoseanwendung eine besondere Priorität. Näheres dazu wird in Kapitel 3 beschrieben.

\footnotetext{
$1 \quad$ M. Sc. Sercan Sade, TH Ostwestfalen-Lippe, Lemgo

2 Prof. Dr.-Ing. Thomas Bartsch, TH Ostwestfalen-Lippe, Lemgo

3 B. Eng. Franziska Albers, TH Ostwestfalen-Lippe, Lemgo

4 M. Sc. Dennis Kowalke, TH Ostwestfalen-Lippe, Lemgo
} 


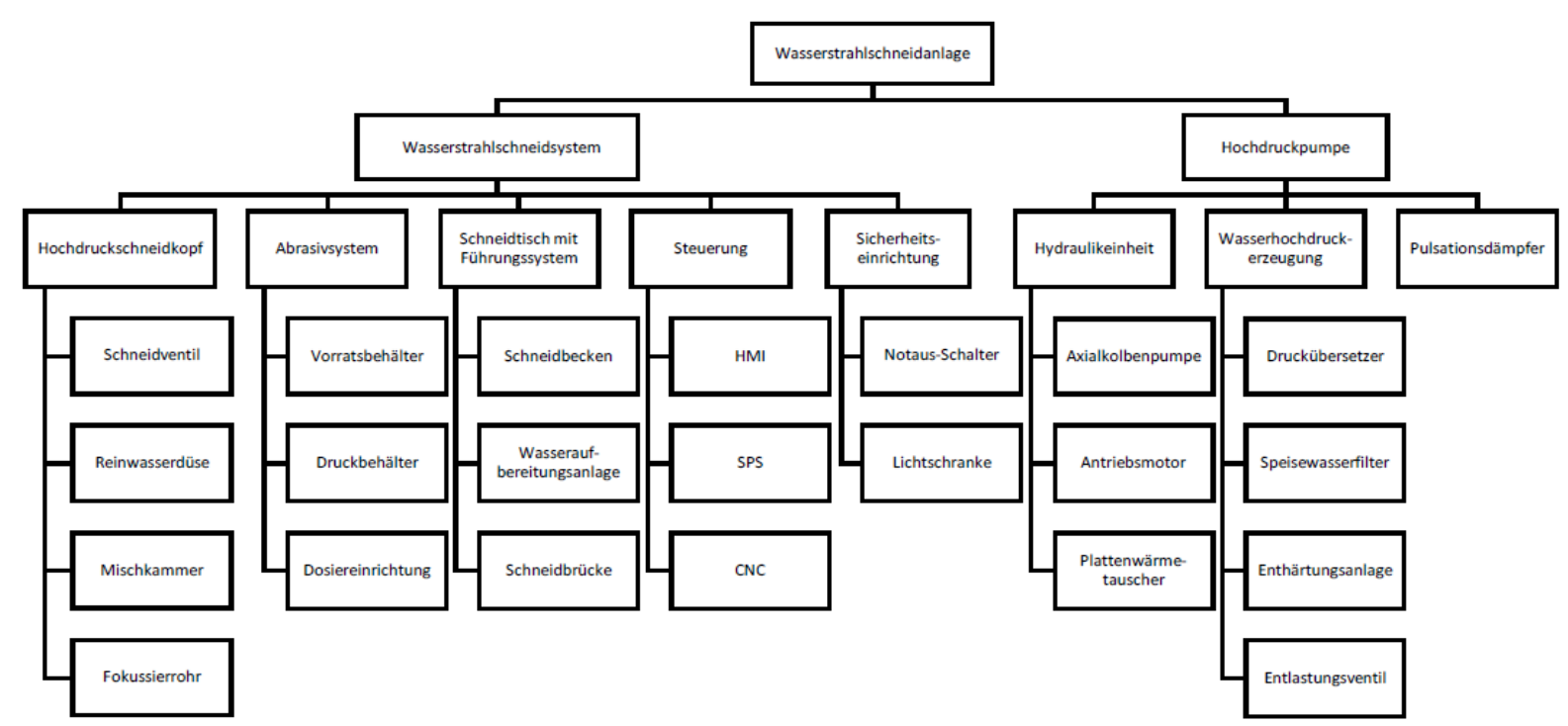

Bild 1: Aufbau der Wasserstrahlschneidanlage

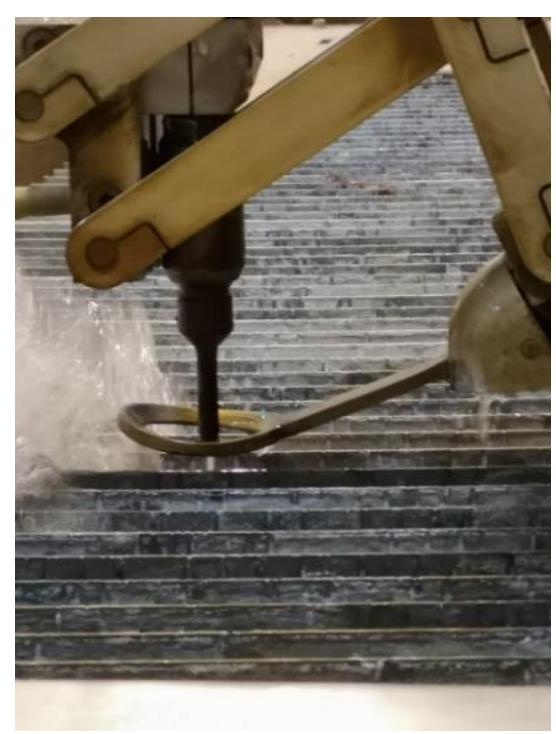

Bild 2: Hochdruckschneidkopf der Wasserstrahlschneidanlage im Betrieb

\section{Instandhaltung}

Um ein tieferes Verständnis dem digitalen Diagnosetool gegenüber zu erzeugen, wird im Vorfeld ein kleiner Einblick in die Grundlagen der Instandhaltung gegeben.

Die DIN 31051 unterteilt die Instandhaltung in drei Grundmaßsnahmen. Diese sind: Wartung, Inspektion und Instandsetzung. Sie dienen dazu einen maximal wirtschaftlichen Betrieb der Anlage zu gewährleisten, indem Störungen und Ausfälle vermieden werden. Eine wichtige Kennzahl bei Maschinen hierfür ist der Abnutzungsvorrat [1]. 
Bild 3 zeigt schematisch den idealen zeitlichen Verlauf des Abnutzungsvorrates einer Anlage.

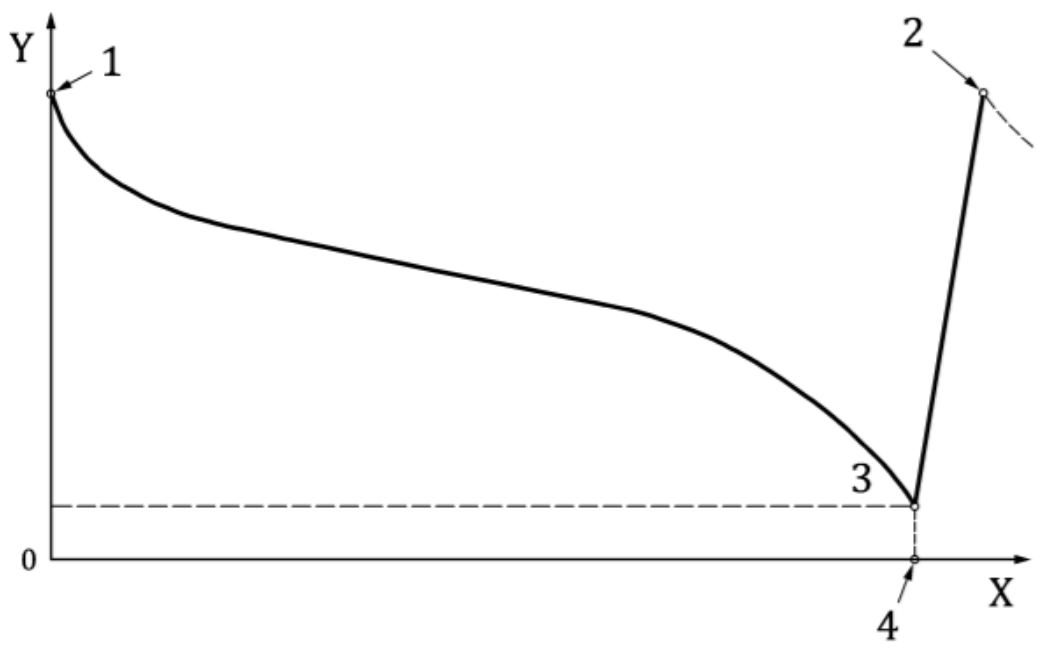

Bild 3: Idealer Verlauf Abnutzungsvorrat [1]

An der Abszisse steht die Zeit X, während die Ordinate den Abnutzungsvorrat Y darstellt. Zum Zeitpunkt $X=0$ befindet sich der Abnutzungsvorrat $Y$ bei 1 . Dies bedeutet eine vollumfängliche Funktionserfüllung ist gewährleistet. Im Laufe der Zeit nimmt der Abnutzungsvorrat ab, bis die Abnutzungsgrenze bei Punkt 3 erreicht ist und es zum Ausfall bei Punkt 4 kommt. Durch fristgerechte Wartung wird das Erreichen der Abnutzungsgrenze verzögert. Die Inspektion dient zur Begutachtung des Istzustandes der Anlage. Diese kann zum Zeitpunkt des Erreichens der Abnutzungsgrenze erfolgen. Es ist jedoch empfehlenswert die Inspektion in regelmäßigen Abständen vor Erreichen dieser Grenze durchzuführen, um einen Ausfall zu vermeiden. Um die Anlage nach dem Ausfall wieder in den funktionsfähigen Zustand bei Punkt 2 zu überführen, bedarf es einer Instandsetzung [1], [5].

Zusammenfassend ergibt sich eine ideale Abnutzung einer Anlage durch eine möglichst lange Abnutzungszeit und einer kurzen Instandsetzungszeit, um die Funktion der Anlage wiederherzustellen. Dieser ideale Verlauf wurde durch die Entwicklung des Diagnosetools der Wasserstrahlschneidanlage angestrebt. Es wurden Werkzeuge und Methoden verwendet, welche den herkömmlichen Instandhaltungsverfahren durch digitale Unterstützung vorauseilen und die erforderliche kurze Instandsetzungszeit fördern. Diese werden im nächsten Kapitel vorgestellt.

\section{Diagnosetool}

Dieses Kapitel bildet den Hauptteil des Beitrages. Es beinhaltet eine Beschreibung des Diagnosetools und konkrete Beispiele. Hierfür wird die digitale Instandhaltung am eingangs erwähnten Hochdruckschneidkopf und an der Hochdruckpumpe der Anlage veranschaulicht.

Für die Umsetzung wurde die Modellierungssoftware „QuickSteps“ genutzt [2]. QuickSteps stellt eine softwarebasierte Methode zur Störfalldiagnose, -behebung und Erfahrungsrückgewinnung in Fertigungsprozessen dar. Die Software besteht aus zwei Teilen: Das Modellierungssystem und das Fehlerdiagnosesystem. Das Modellierungssystem wird verwendet, um Projekte in QuickSteps zu erstellen und zu bearbeiten. Das Fehlerdiagnosesystem dient zur Störfallbestimmung und zur Ausgabe von entsprechenden Handlungsanweisungen [2].

Es existieren also zwei Schnittstellen zwischen Mensch und Diagnosetool. Zum einen zu der Modellieroberfläche und zum anderen in das Fehlerdiagnosesystem, welches zur operativen Instandhaltung an der Anlage genutzt wird. Beide Schnittstellen werden im Folgenden näher erläutert. 


\subsection{Modellierungssystem}

Im Modellierungssystem wurde zunächst das digitale Instandhaltungstool entwickelt. Es wurden alle bekannten in Betracht kommenden Handlungsmaßnahmen digital erfasst und in einer Baumstruktur (Abb. 4) abgebildet. Die einzelnen Elemente enthalten Seiten, bei denen dem Bediener der Wasserstrahlschneidanlage unterstützende Anweisungen gegeben werden. Die Quellen für die Anweisungen stammen zu einem Teil aus den Handbüchern des Herstellers und wurden zum anderen Teil um das Erfahrungswissen von Mitarbeitern ergänzt. Bild 4 zeigt die Baumstruktur der Diagnoseanwendung. Die Instandhaltungsmaßnahmen und unterstützenden Anweisungen sind Gegenstand des Fehlerdiagnosesystems.

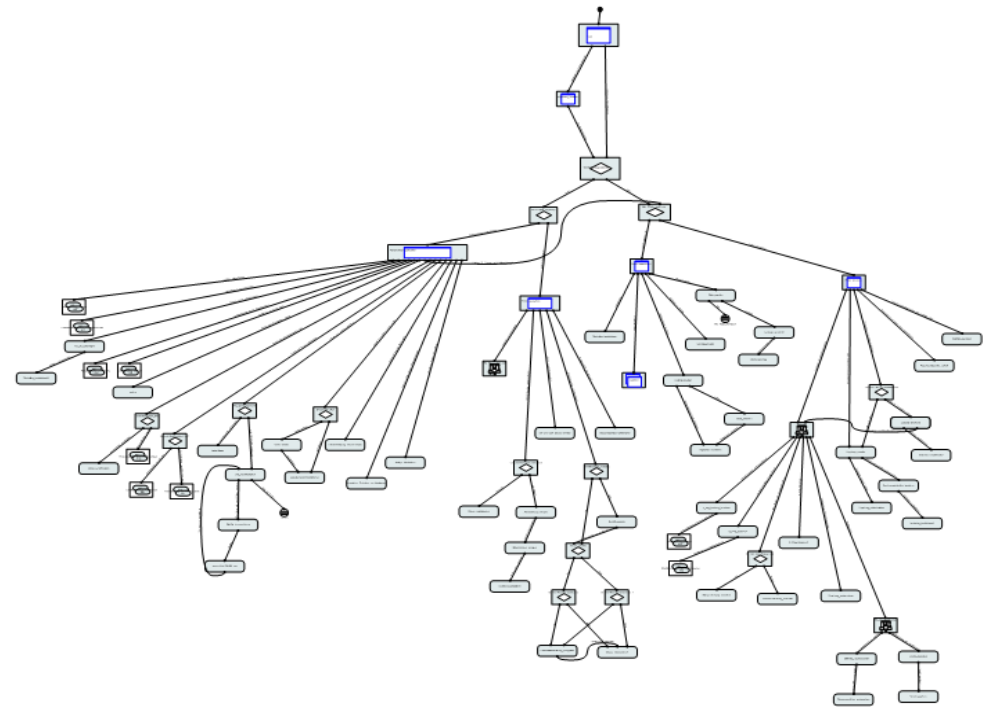

Bild 4: Baumstruktur des Modellierungssystems

In Bild 5 wird ein Einblick in die Entstehung der Baumstruktur und in einige wichtige Kernelemente des Anwendungsprogrammes gegeben.

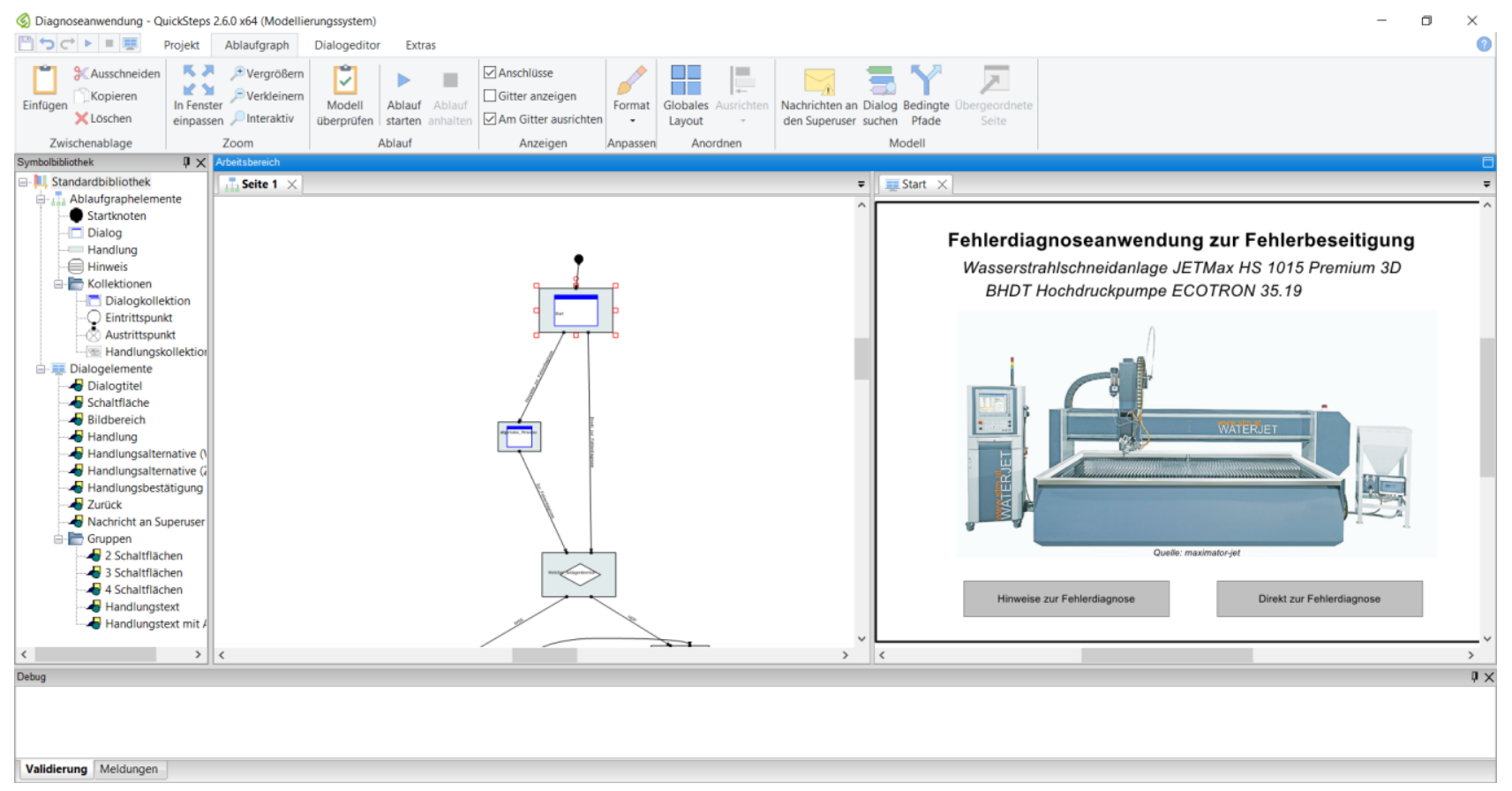

Bild 5: Modellieroberfläche QuickSteps 
In der Modellierungsoberfläche existieren zwei Fenster. In dem ersten entwickelt der Anwender die Baumstruktur (links in Bild 5). Das zweite Fenster (rechts in Bild 5) wird mit Information und Erfahrungswissen angereichert, welches das Fehlerdiagnosesystem bildet und an der Anlage zum Einsatz kommt.

Jedes QuickSteps-Modell beginnt mit einem Startknoten. Dieser ist als schwarzer Punkt in Bild 5 dargestellt. Auf diesen folgt der wichtigste Baustein in der Modellierungsumgebung, der Dialogbaustein. Der erste Dialogbaustein wird auch Einstiegsdialog genannt. Die Modellierung des Einstiegsdialoges wird in Bild 5 veranschaulicht. Bei diesem wird dem Instandhalter zunächst eine Einführung in das digitale Instandhaltungswerkzeug geboten. Über die zwei grauen Schaltflächen hat dieser die Möglichkeit die nächsten Schritte einzuleiten.

Das Diagnosetool wurde so aufgebaut, dass es dem Instandhalter der Wasserstrahlschneidanlage für Maßßnahmen der Wartung, Inspektion und Instandsetzung unterstützend zur Verfügung steht. In Bild 6 wird die Entstehung der Baumstruktur verdeutlicht. Von dem Dialogbaustein „Fehlerursache benennen“ führen mehrere Kanten zu den Blättern des Baums. Die Blätter des Baums bilden die letzte Instanz der Baumstruktur. Sie beinhalten in diesem Fall die konkreten Handlungsanweisungen zur Fehlerbeseitigung [3].

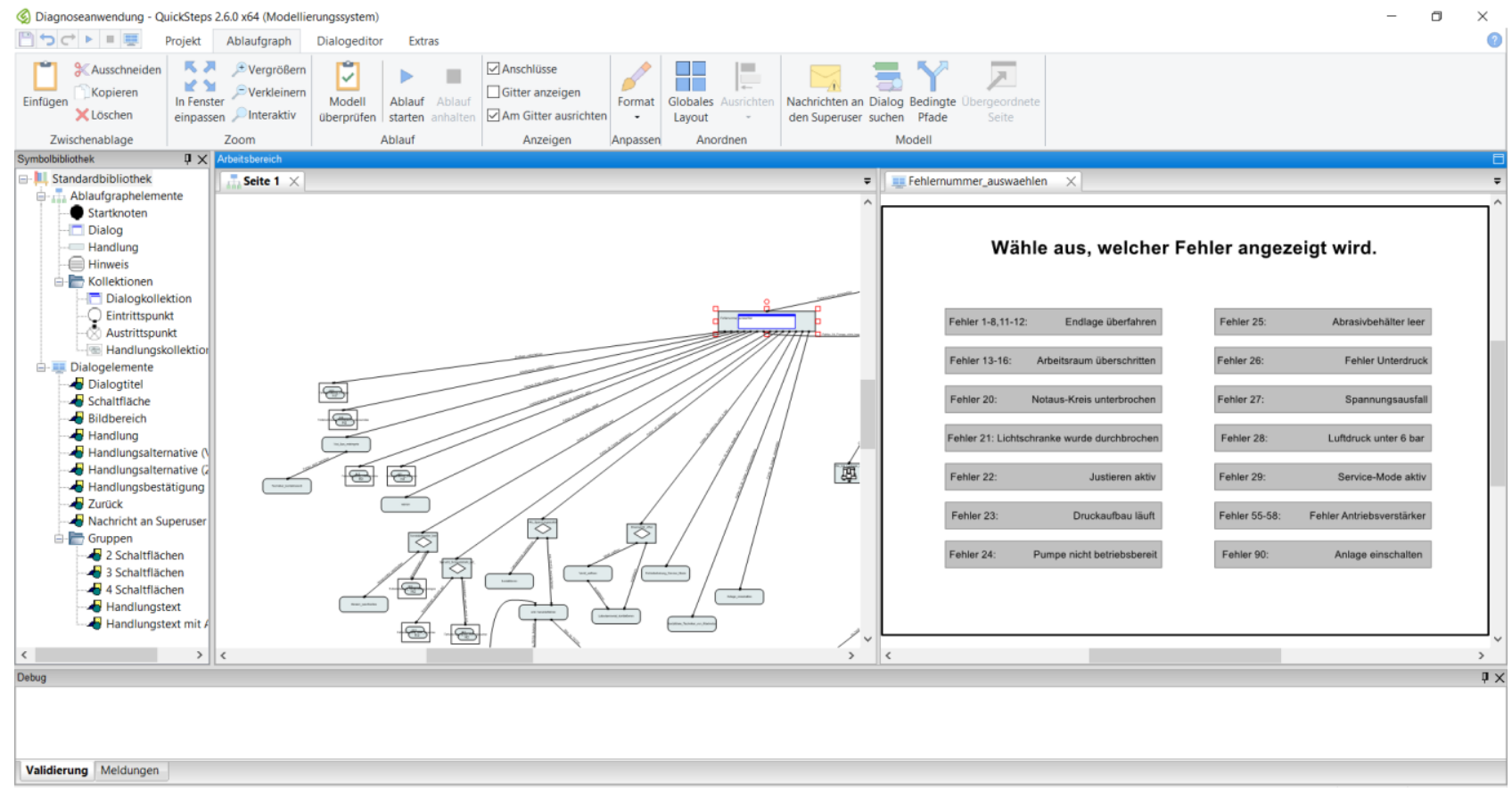

Bild 6: Fehlerbehebung von angezeigten Fehlern

Ein Teil der Diagnoseanwendung setzt sich mit der Behebung von Fehlern auseinander, die am Bedienerterminal der Wasserstrahlschneidanlage angezeigt werden. Der Anlagenbediener hat so die Möglichkeit gezielt seinen Fehler auszuwählen und wird je nach ausgewählter Schaltfläche zu entsprechenden Handlungsmaßnahmen, die zur Entstörung dienen, weitergeleitet.

Bei einigen Schaltflächen wird der Anlagenbediener durch sogenannte Dialogkollektionen geleitet. Der Unterschied zum bereits erwähnten Dialog besteht darin, dass die Dialogkollektion mehrere Dialoge beinhaltet. Eine Fehlernummer öffnet beispielsweise eine Dialogkollektion, welche mehrere Handlungsanweisungen enthält, die sequentiell abgearbeitet werden, um den funktionsfähigen $\mathrm{Zu}-$ stand der Anlage wiederherzustellen. Diese Methode wurde genutzt, um die Baumstruktur aus Bild 4 übersichtlich zu gestalten. 


\subsection{Fehlerdiagnosesystem}

Während sich das Modellierungssystem als sehr entwicklerorientiert herausgestellt hat, bildet das Fehlerdiagnosesystem die Komponente, welche für den Bediener und Instandhalter bei der operativen Tätigkeit an der Anlage wichtig ist. Es wird idealerweise auf einer grafischen Oberfläche (GUI) direkt an der Maschine bereitgestellt. In Bild 7 wird der vom Entwickler modellierte Einstiegsdialog aus der Fehlerdiagnosesicht gezeigt.

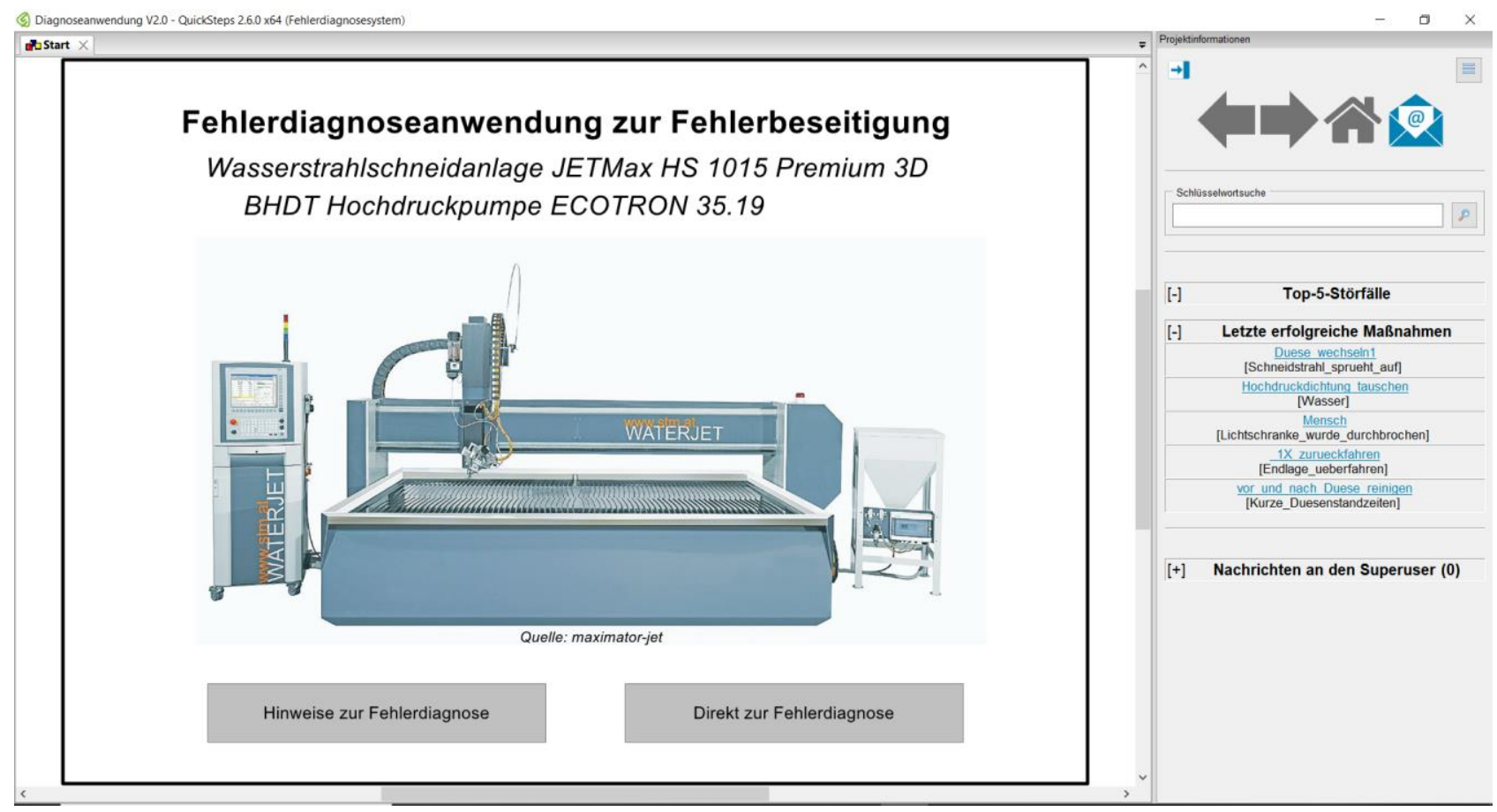

Bild 7: Einstiegsdialog Diagnoseanwendung

Es wird erkennbar, dass das Fehlerdiagnosesystem einen anderen Schwerpunkt hinsichtlich der Funktionalität hat. Die Oberfläche bietet dem Anwender die Möglichkeit über die Schaltflächen von der Fehlerursache bis hin zur Störungsbeseitigung digital unterstützt zu werden. Außerdem besteht die Option Störfälle statistisch zu Qualitätssicherungszwecken zu erfassen und auszuwerten.

Im Rahmen der Entwicklung der Diagnoseanwendung wurden auch Methoden und Werkzeuge verwendet, welche dem Bediener bei komplexen Instandhaltungsaufgaben unterstützen. Eines davon ist der sogenannte Bilddialog. Ein Beispiel aus der Diagnoseanwendung wird in Bild 8 gezeigt. 


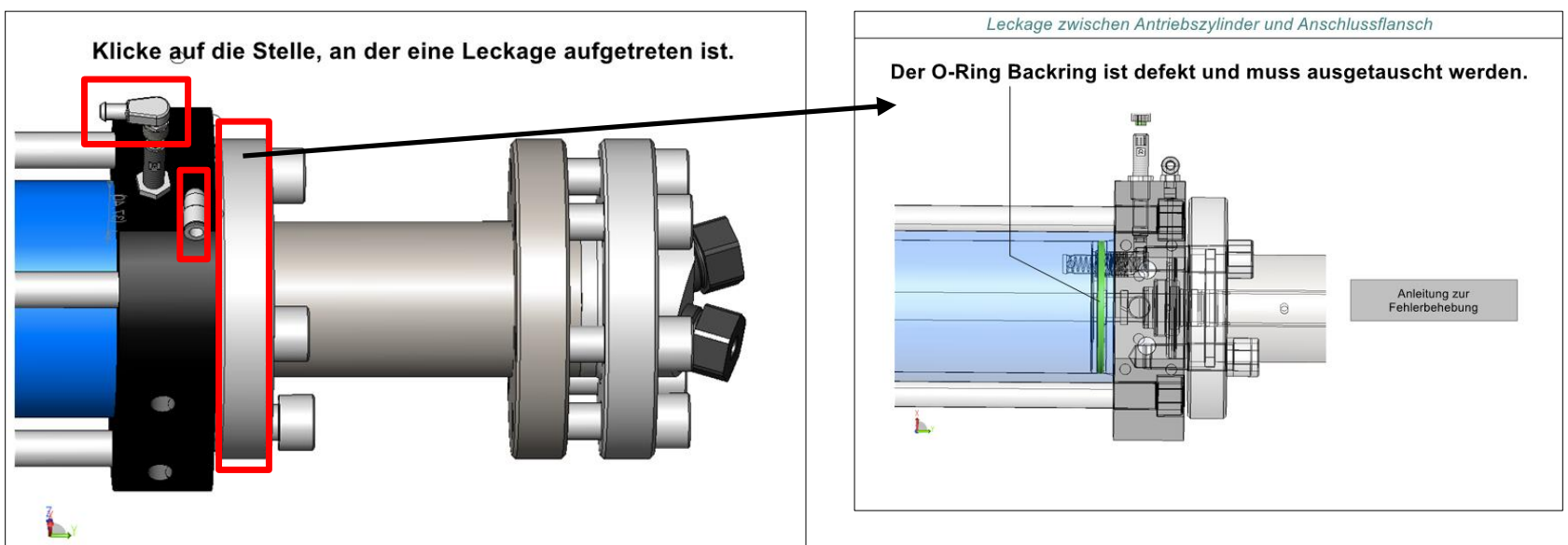

Bild 8: Bilddialog zur Leckagebehebung

Im Vergleich zu einer Dialogkollektion beinhaltet der Bilddialog keine Schaltflächen, die den Instandhalter zu den durchzuführenden Maßnnahmen führen. Diese erscheinen erst, sobald er mit seiner Hand die jeweilige Stelle im Bilddialog berührt. Bild 8 zeigt die Hochdruckpumpe der Wasserstrahlschneidanlage. Die Pumpe ist anfällig für diverse Leckagen an den Anschlüssen oder Flanschen. Die möglichen Leckagestellen sind rot umrandet, sodass dem Bediener der Diagnoseanwendung direkt übersichtlich dargestellt wird, welche anwählbar sind. Je nach getroffener Wahl folgen entsprechende Handlungsanweisungen.

In Bild 9 wird eine weitere Methode gezeigt, die zur effizienten Instandhaltung befähigt. Es handelt sich hierbei um die Demontage des Hochdruckschneidkopfes, die in regelmäßigen Abständen zu Wartungszwecken erforderlich ist. Um eine Gewindeschädigung des Schneidkopfes zu vermeiden, wurde die Demontage auf einem Video aufgezeichnet, welches sich öffnet, wenn die entsprechende Schaltfläche angewählt wird.

So hat der Monteur die Möglichkeit direkt an der Anlage den Demontageprozess vorher anzuschauen, um so kritische Montagefehler zu umgehen.

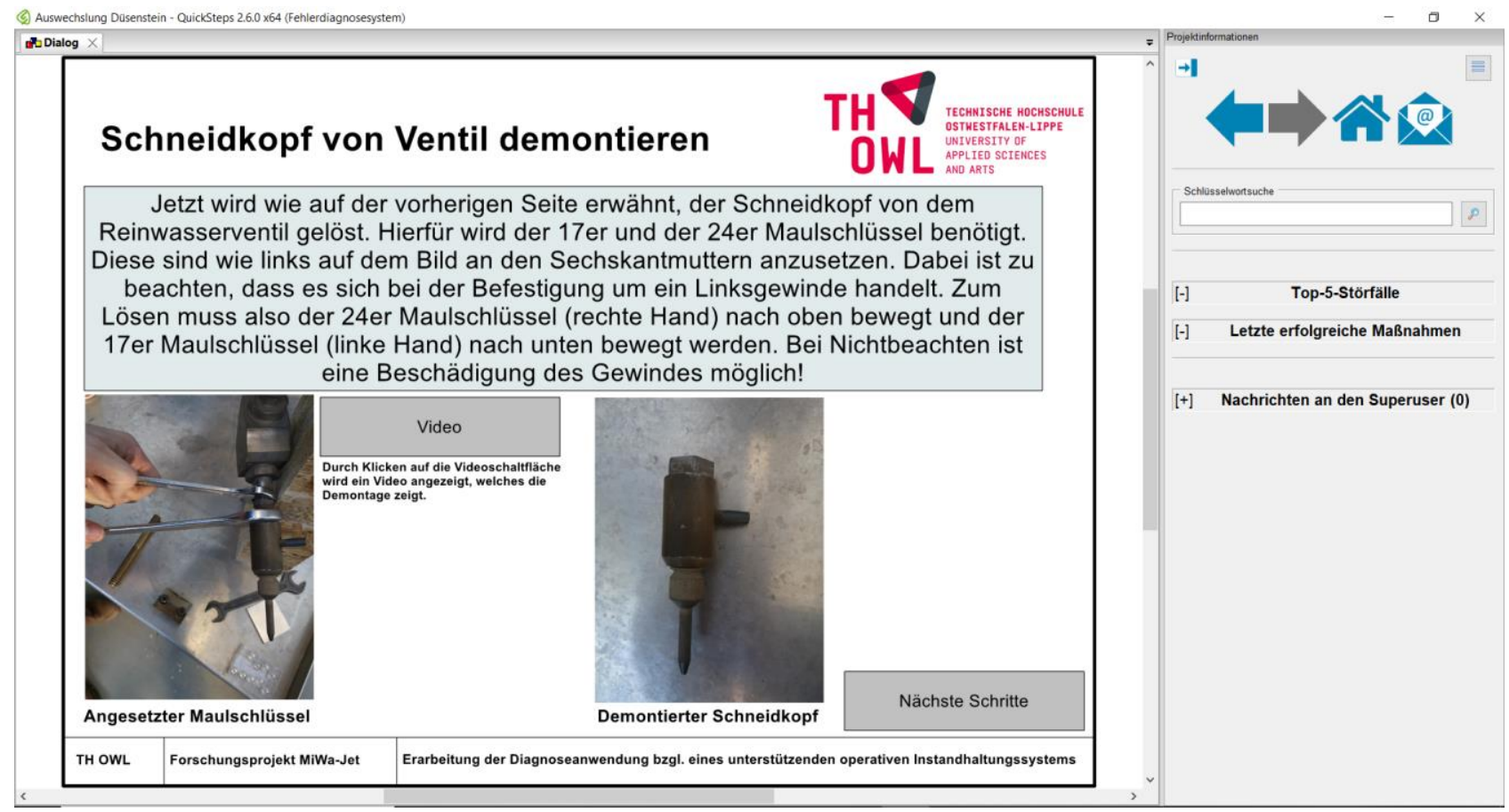

Bild 9: Demontage Hochdruckschneidkopf 


\section{Fazit}

Mit der Diagnoseanwendung ist es gelungen, ein digitales interaktives Instandhaltungstool zu entwickeln, welches den Instandhaltungsprozess der Wasserstrahlschneidanlage verbessert hat. Durch die schnelle Verfügbarkeit und Zugänglichkeit von konkreten Handlungsmaßnahmen wird ein gesunkener Abnutzungsvorrat der Wasserstrahlschneidanlage zügig wiederhergestellt.

Das Tool bietet eine umfangreiche Unterstützung für Personen, die an der technischen Anlage Instandhaltungsmaßnahmen durchführen. Es leitet ebenfalls dazu an, auftretende Fehler zu ungünstigen Zeitpunkten, wie z. B. an Wochenenden oder Feiertagen, ohne die Hilfe einer Fachkraft zu beheben und den funktionsfähigen Zustand der Anlage wiederherzustellen.

Einen weiteren großen Mehrwert, den die Diagnoseanwendung bietet ist, dass sie nicht nur im operativen Geschäft nutzbar ist, sondern auch für strategische Qualitätsverbesserung geeignet ist [4]. Durch die Auswertung der Störfälle ist es möglich, häufig auftretende Fehler genauer zu analysieren und situationsabhängige Verbesserungspotenziale abzuleiten.

\section{Förderhinweis}

Das Forschungsprojekt wird gefördert durch das Bundesministerium für Wirtschaft und Energie.

\section{Literatur}

[1] DIN 31051. Grundlagen der Instandhaltung (Juni 2019).

[2] Gesellschaft zur Förderung angewandter Informatik e. V. (GFaI) (2020). QuickSteps Dokumentation. Berlin.

[3] URL: https://www.itwissen.info/Baumstruktur-tree-structure.html (abgerufen am 05.12.2021).

[4] DIN EN ISO 9001. Qualitätsmanagementsysteme - Anforderungen (November 2015).

[5] Reichel, J., Müller, G. \& Mandelartz, J. (2009). Betriebliche Instandhaltung. Berlin-Heidelberg: Springer-Verlag. 